Hard x-ray bursts observed in association with Rayleigh-Taylor instigated current disruption in a solar-relevant lab experiment

R. S. Marshall, M. J. Flynn, and P. M. Bellan

Citation: Physics of Plasmas 25, 112101 (2018); doi: 10.1063/1.5054927

View online: https://doi.org/10.1063/1.5054927

View Table of Contents: http://aip.scitation.org/toc/php/25/11

Published by the American Institute of Physics

\section{PHYSICS TODAY}

MANACER'S GUIDE

WHITEPAPERS
READ NOW

PRESENTED BY Accelerate R\&D with
Multiphysics Simulation 


\title{
Hard x-ray bursts observed in association with Rayleigh-Taylor instigated current disruption in a solar-relevant lab experiment
}

\author{
R. S. Marshall, M. J. Flynn, and P. M. Bellan \\ Applied Physics and Materials Science, California Institute of Technology, Pasadena, California 91125, USA
}

(Received 5 September 2018; accepted 14 October 2018; published online 1 November 2018)

\begin{abstract}
Measurements by multiple X-ray detectors show transient emission of a $1 \mu$ s pulse of non-monoenergetic $\sim 6 \mathrm{keV}$ X-rays by a cold, dense MHD-driven plasma jet. Because the collision mean free path is much smaller than the jet dimensions, the acceleration of particles to high energy was not expected. The X-ray pulse occurs when the jet undergoes a kink instability which accelerates the jet laterally so that a fast-growing secondary Rayleigh-Taylor instability is triggered which then breaks the jet. The jet breaking is correlated in time with several other fast changing phenomena. It is proposed that despite the short collision mean free path, an inductive electric field associated with this breaking accelerates a certain subgroup of electrons to $\mathrm{keV}$ energies without any of these electrons undergoing collisions. It is further proposed that after being accelerated to high energy, these fast electrons are suddenly decelerated via collisions and radiate X-rays. Published by AIP Publishing. https://doi.org/10.1063/1.5054927
\end{abstract}

\section{INTRODUCTION}

Acceleration of charged particles to energies orders of magnitude larger than the ambient thermal energy is a welldocumented but a mysterious feature of laboratory, solar, and astrophysical plasmas. Examples are numerous and start with the 60 year old observation in the Zero Energy Thermonuclear Assembly (ZETA) device of neutrons ${ }^{1}$ initially interpreted as the by-product of thermonuclear fusion reactions. However, doubt was quickly cast over this interpretation as the ZETA plasma was shown to be only $10-10^{2} \mathrm{eV}$, much colder than the required $10^{4} \mathrm{eV}$ temperature for thermonuclear fusion. ${ }^{2-5}$ More recent laboratory examples include dense plasma focus devices which use a transient magnetized plasma to produce small quantities of neutrons ${ }^{6,7}$ presumably by the same mechanism that occurred in ZETA. A transient burst of X-rays was observed in association with Spheromak formation and was believed to be associated with the pinching off of the plasma from the gun electrodes. ${ }^{8}$ Other magnetic confinement devices such as tokamaks produce super-thermal particles as well. ${ }^{9}$ Examples in nature also occur and include solar flares which are transient, localized solar corona events that produce electrons with energies up to $10^{8} \mathrm{eV}$ and ions up to $10^{9} \mathrm{eV}$ even though the corona is only $10^{2} \mathrm{eV}$ or less. ${ }^{10-14}$ High energy non-thermal particles and energetic photons observed in astrophysical jets provide yet another example of production of super-thermal particles in a natural environment. ${ }^{15,16}$

The common factors in these very different regimes are that (i) charged particles are accelerated to energies orders of magnitude larger than thermal, (ii) the process is transient, (iii) magnetic fields and electric currents are involved, and (iv) there appears to be some sort of instability. Mechanisms such as runaway ions in small regions, ${ }^{17}$ creation of a deuterium beam, ${ }^{3}$ wave-particle resonance, ${ }^{11}$ stochastic motion, ${ }^{14}$ and Fermi acceleration ${ }^{18}$ were previously proposed, but magnetic reconnection is now thought to play a crucial role. ${ }^{19-21}$
Since the accelerated particles are significantly more energetic than the thermal particles, the energy reservoir powering this acceleration is unlikely to come from the thermal particles and instead is presumed to come from the energy stored in the magnetic field being released through magnetic reconnection.

It is not known why only a subset of particles are energized, how this subset is selected, and why this subset can be accelerated in an extremely collisional plasma. We report two striking experimental observations providing new insights into this process: (i) short transient hard X-ray bursts are consistently observed by multiple detectors viewing a very cold, highly collisional, dense laboratory plasma jet and (ii) a magnetic probe array measures an abrupt change in the magnetic topology of the system. Both observations correlate in time with a kink-instigated Rayleigh-Taylor (RT) instability that breaks the plasma jet apart. The analysis of experimental measurements indicates that the X-rays are likely from Bremsstrahlung of a tiny subset of electrons accelerated by a large inductive electric field in the breaking region. This detection of hard X-rays emitted from a cold, dense collisional plasma is remarkable because one would expect the high collisionality to preclude acceleration of any particles to high energies. The proposed mechanism is simpler than the previously suggested mechanisms of Fermi acceleration, shock acceleration, wave-particle resonance, stochastic heating, or exceeding the Dreicer runaway electric field. This simplicity should make the process widely relevant.

This paper is organized as follows: Sec. II summarizes the experimental parameters and sequence. Section III describes the X-ray detectors and measurement. Section IV reports magnetic field measurements and shows that the form of the field after breaking differs substantially from the form before. Section V lists eight simultaneous, distinct phenomena taking place when the jet breaks. Section VI compares the available energy sources with the energy in the X-ray burst and estimates the electric field strength from the observed X-ray energy and the physical dimensions of the breaking region. 
Section VII discusses the underlying physical origin for the Xray burst and for all eight simultaneous phenomena. Section VIII contains the conclusion.

\section{EXPERIMENTAL CONFIGURATION}

The Caltech jet experiment produces a cold, dense, collisional low- $\beta$ MHD-driven jet with an initial radius of a few $\mathrm{cm}$ and a length increasing to several 10's of $\mathrm{cm}$ in 30-50 $\mu \mathrm{s}$. As shown in Fig. 1, the jet is created in a $1.4 \mathrm{~m}$ diameter, $1.6 \mathrm{~m}$ long vacuum chamber which has a pair of coplanar, concentric copper electrodes mounted on one end. The inner disk electrode is connected to a capacitor bank, and the outer annular electrode is connected directly to the ground allowing for a potential difference of $3-6 \mathrm{kV}$ to be applied across the electrodes during discharge. The experimental setup and shot firing process have been described in detail elsewhere ${ }^{22-24}$ and will be summarized here. First, a coil behind the electrodes is energized, creating a dipole-like poloidal magnetic field that links the inner and outer electrodes. Fast gas valves then open and puff a gas cloud into the vacuum chamber through eight radially oriented, equally spaced concentric pairs of holes on the inner and outer electrodes. After gas injection, the 120 microfarad capacitor bank establishes a large potential difference $(3-6 \mathrm{kV})$ across the electrodes. The applied voltage ionizes the gas cloud in front of the electrodes along the poloidal magnetic field lines from the coil. Electric current driven by the discharging capacitor bank flows along the eight plasma arches. Hydrogen, nitrogen, argon, and krypton gases can be used. A pulse forming network (PFN) is used to sustain the jet for up to $50 \mu \mathrm{s}$.

Spectroscopic line ratios indicate nominal $2 \mathrm{eV}$ electron temperatures, while Doppler broadening of spectral lines

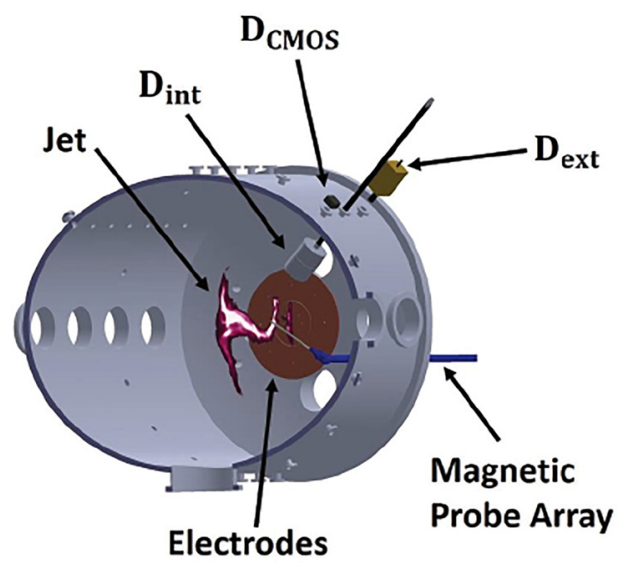

FIG. 1. Experimental layout: the electrodes are shown in the center in brown. The magnetic probe array can both move axially and rotate and is shown oriented with the array spanning the center of the electrodes. $D_{C M O S}$ is the small, black rectangular camera housing on the third flange from the end. $D_{i n t}$ is mounted next to it on the second flange; its scintillators are housed in the cylindrical body inside the vacuum chamber, and the scintillated photons are transmitted through optical fibers coming out of the black tube through the flange. $D_{e x t}$ is shown on the flange farthest to the right; it was operated on the second flange currently home to $D_{\text {int }}$ prior to its existence. For the drawing, $D_{\text {ext }}$ was moved one flange over. $D_{\text {amptek }}$ was operated on the far right flange where $D_{\text {ext }}$ is shown. An Imacon 200 high-speed camera is positioned far off to the right and photographs through one of the large side windows. indicates similar ion temperatures. ${ }^{22}$ A laser interferometer ${ }^{25}$ indicates a nominal density $n_{e} \simeq 3 \times 10^{22} \mathrm{~m}^{-3}$, giving a nominal $0.6 \mu \mathrm{m}$ electron collision mean free path using the nominal temperature of $T=2 \mathrm{eV}$. A $Z=2$ ionization state is assumed based on spectroscopic measurements at the time of the X-ray burst. A set of images showing certain key points in the jet's life cycle is shown in Fig. 2. A frame rate of $10^{6}$ frames per second (FPS) was used to capture these images. When the jet reaches a critical length at which the KruskalShafranov ideal MHD kink threshold is crossed, a fast growing kink instability develops and causes the jet to become helical. ${ }^{24,26}$ The radially outward acceleration associated with the exponential growth of the helical instability triggers a Rayleigh-Taylor (RT) secondary instability ${ }^{23}$ which chokes down the jet diameter at a sequence of short wavelength interchange ripples. The kink is the large arch in Fig. 3(a), and the RT instability is the set of ripples on the inboard side of the arch. The electron and ion temperatures increase to 6 $\mathrm{eV}$ and $16 \mathrm{eV}$, respectively, at the time and approximate location of the RT instability. ${ }^{22}$ High-speed imaging shows that argon jets break up when the RT occurs as seen in the image in Fig. 3(b), whereas hydrogen jets do not. ${ }^{23}$ This differing behavior is interpreted as RT-instigated magnetic reconnection resulting from the RT instability choking down the current cross-section to be smaller than the ion skin depth $c / \omega_{p i}$ in the argon jet as indicated in Fig. 3(b) but not in the hydrogen jet which has a much smaller ion skin depth. ${ }^{23}$

\section{X-RAY BURST AND DETECTOR DETAILS}

Four different detectors have been used to detect X-ray emission from the jet. These are denoted as follows: (i) $D_{\text {ext }}$, a single-channel plastic scintillator mounted outside the chamber, (ii) $D_{\text {int }}$, a vacuum-tight 7-channel plastic scintillator detector mounted inside the vacuum chamber, (iii) $D_{\text {amptek }}$, a commercial Amptek XR100 Silicon Drift Detector mounted outside the chamber, and (iv) $D_{C M O S}$, a windowless CMOS camera mounted outside the chamber. The caption of Fig. 1 indicates how the detectors are positioned. $D_{\text {ext }}, D_{\text {int }}$, and $D_{\text {Amptek }}$ have time resolution with minimal energy resolution, while $D_{C M O S}$ has energy resolution but no time resolution. The data from these four detectors show that a short $\sim 10^{-6} \mathrm{~s}$ burst of non-mono-energetic X-rays is detected between $t \approx 24$ and $40 \mu$ s at the same time as several other distinct transient phenomena.

\section{A. External detector, $D_{\text {ext }}$}

The one inch diameter cylindrical plastic scintillator in $D_{\text {ext }}$ is wrapped by aluminum foil and then by black electrical tape to block visible light. A $2.5 \mathrm{~m}$ light pipe carries scintillated blue photons from the tapered end of the scintillator to a photomultiplier tube inside a highly shielded box. The photomultiplier is powered by batteries to prevent ground loops. The electrodes are outside the field of view of $D_{\text {ext }}$ and so cannot be the source of the X-rays, i.e., the X-rays must originate in the plasma.

Figure 4 shows a sample dataset. The X-ray data from $D_{\text {ext }}$ are shown in blue, and the voltage across the electrodes measured by a high voltage probe is shown in black. At 


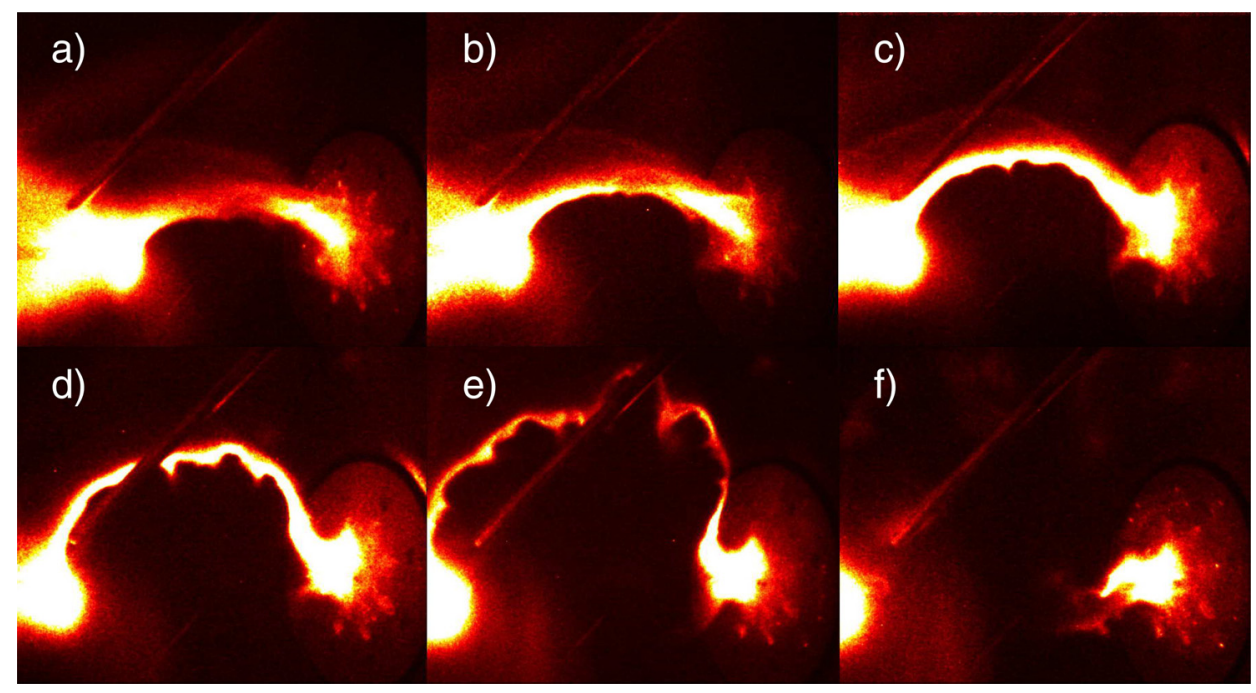

FIG. 2. Six false color images taken by the ultra-high-speed camera capturing at $10^{6}$ frames per second from shot 18758, an Argon jet. The first image (a) is $25 \mu$ s into the shot, and each subsequent image is $1 \mu$ s later. (a) and (b) show a kinked plasma that is accelerating radially outward. (c) and (d) show fast-growing Rayleigh-Taylor ripples superposed on the kinking arch. From (c) to (e), the ripples get larger, and in the microsecond between (e) and (f), the ripples get so large that the plasma breaks apart.

$t \approx 26.5 \mu \mathrm{s}, D_{\text {ext }}$ detects an X-ray burst and a nearly simultaneous $500 \mathrm{~V}$ jump appears across the electrodes.

The X-ray energy is estimated using $D_{\text {ext }}$ data from 75 consecutive shots with varying thicknesses of aluminum foil placed in the X-ray path. To establish a reference, 20 consecutive shots were taken with no foil (other than the foil
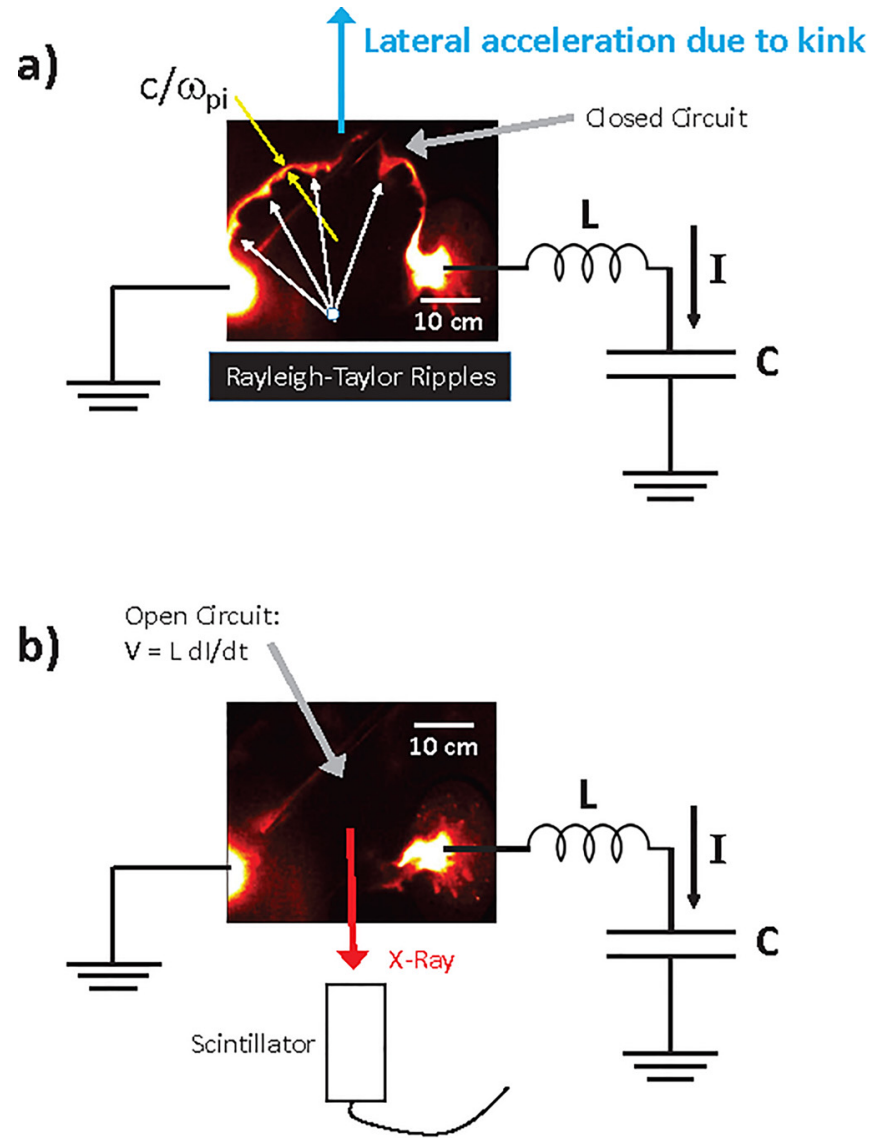

FIG. 3. Images of the argon plasma just before and after the RT instability in a circuit diagram indicating the power electronics. (a) shows the plasma at $t=29 \mu$ s into shot 18758 as a closed circuit when the RT instability has just started. The plasma is undergoing lateral acceleration caused by the kink instability. The RT ripples are indicated by the white arrows. (b) shows the plasma and circuit $1 \mu \mathrm{s}$ later at $t=30 \mu \mathrm{s}$, just after the RT instability has broken the jet to form an open circuit. A voltage $L d I / d t$ appears across the gap. wrapping the scintillator). Then, 20, 10, 20, and 5 shots were taken with $86,124,173$, and $297 \mu \mathrm{m}$ of additional attenuating foil, respectively. Transmission fractions for the varying thicknesses of foil were the least-squares fit with known aluminum transmission data as a function of energy. ${ }^{27}$ The best fit was $5.8 \mathrm{keV}$ with $4.3 \mathrm{keV}$ lower bound and $6.4 \mathrm{keV}$ upper bound; this fitting is shown in Fig. 5 . The $D_{\text {ext }}$ data by itself do not provide sufficient information to tell whether the $\mathrm{X}$ rays are mono-energetic or not. Secs. III B and III D will discuss this in detail.
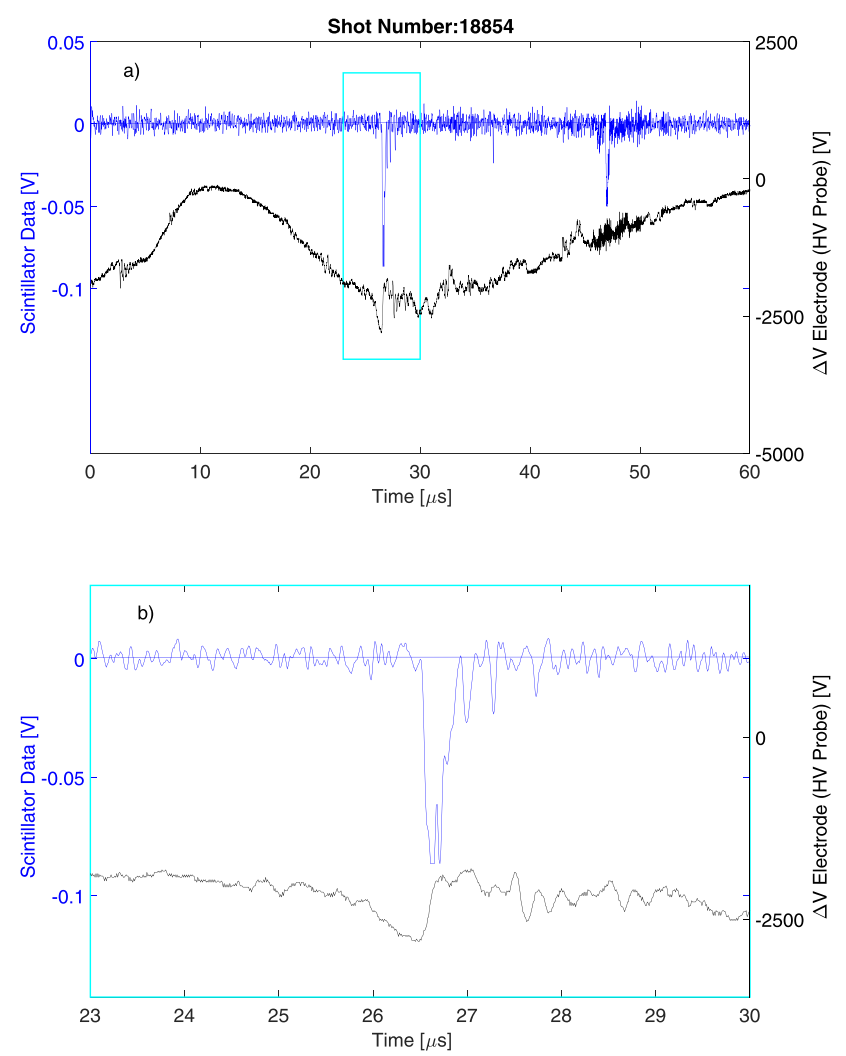

FIG. 4. $D_{\text {ext }}$ X-ray signal (top, blue trace) and high voltage probe signal (bottom, black trace) from shot 18854. The $\Delta \mathrm{V}$ electrode is the voltage across the electrodes measured using a high voltage probe. The very short $\mathrm{X}$-ray signal is coincident with a jump in the high voltage probe measurement. (a) shows the full trace of both diagnostics. (b) is zoomed in for times $t=23-30 \mu$ s bounded by the cyan box in (a). 


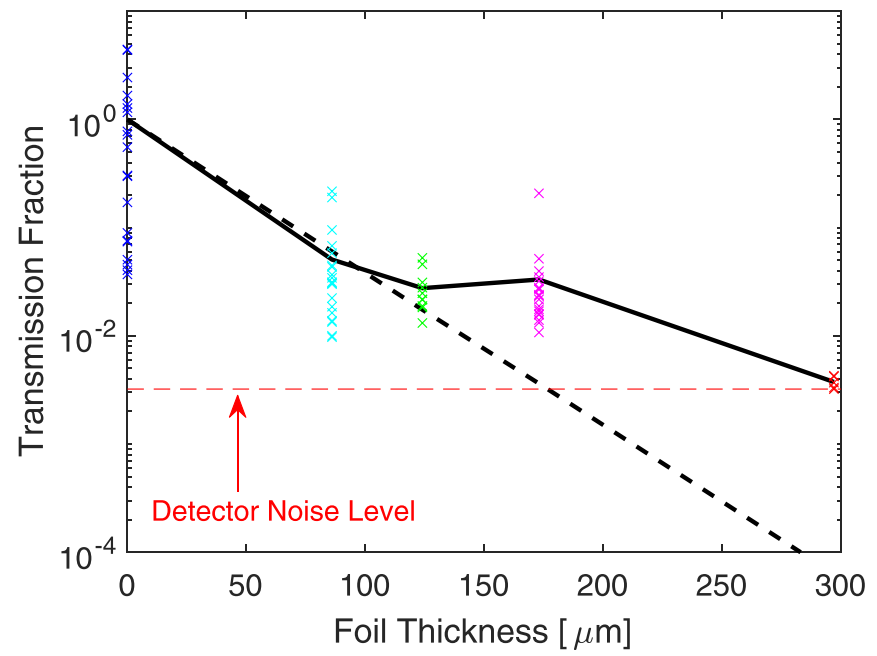

FIG. 5. Plot shows the exponential attenuation of the X-ray signal measured by $D_{\text {ext }}$ with the increasing aluminum foil thickness on a logarithmic vertical axis. Each " $x$ " is a measured signal amplitude normalized to the average amplitude of the 20 measurements taken with no added attenuating foil. Signals were allowed to saturate to increase the dynamic range in the more sensitive measurements, and as a result, some did saturate when no added foil was used. Signal strengths for saturated signals were estimated by fitting their pedestals to the pedestal of a scaled signal with a known strength. The average signal transmission for the number of shots taken with each thickness of the aluminum foil is shown in black and used to calculate the best fit, shown as the black dashed line. The red data points and dashed line represent the noise in the system and help to explain why the best fit diverges at a high thickness. The noise is calculated as the standard deviation of the data for the $297 \mu \mathrm{m}$ foil thickness case where no X-rays were observed above the noise. The best fit is calculated via least-squares using data from Henke et al. ${ }^{27}$

The histogram in Fig. 6 shows that the X-ray burst appears to happen randomly between 24 and $40 \mu \mathrm{s}$. When images from the high-speed framing camera and probe measurements of the electrode voltage are compared to the timing of the X-ray pulse, a striking correlation becomes readily apparent. The X-ray pulse does not occur randomly but instead correlates with both the visual observation of the plasma undergoing the RT instability breaking the jet and also a transient jump of the electrode voltage. Figure 7 shows this correlation between the times of the X-ray signal, the

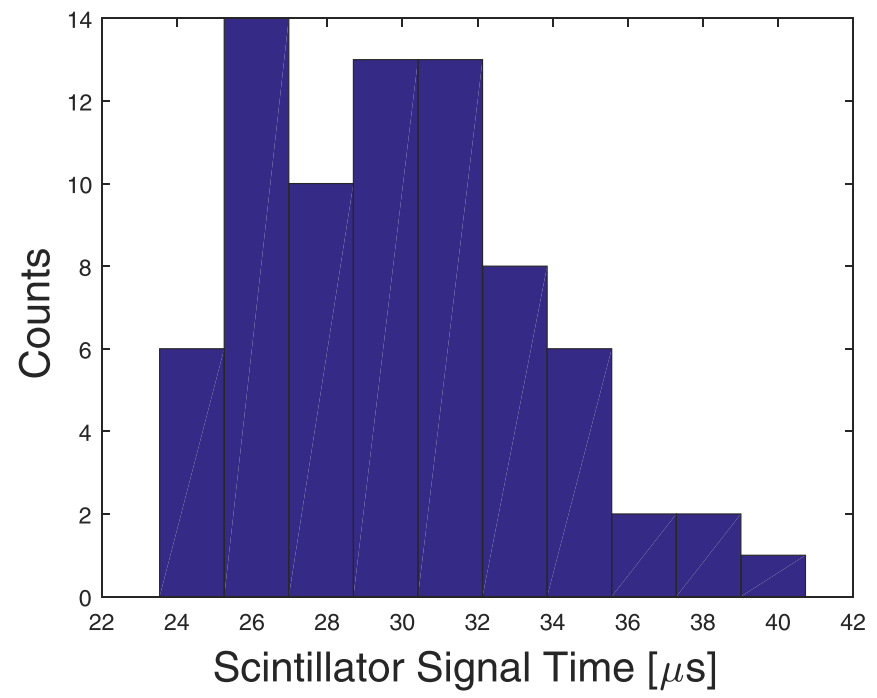

FIG. 6. Histogram of the times at which the X-ray signal is observed by $D_{\text {ext }}$.
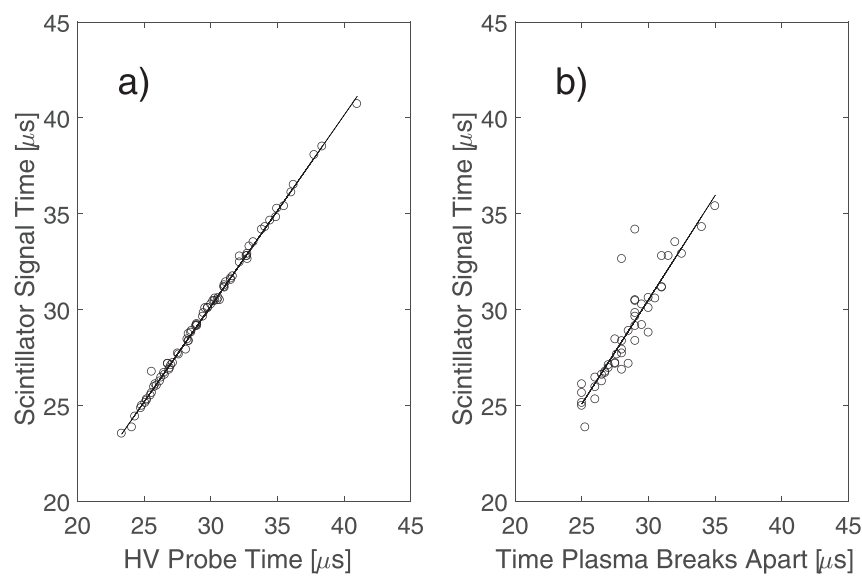

FIG. 7. (a) shows the timing of the fast jump in the HV probe (horizontal axis) versus the timing of the X-ray signal measured by $D_{\text {ext }}$ (vertical axis), showing that the X-ray is coincident with the high voltage jump. (b) shows the time when the plasma breaks apart from the RT instability (horizontal axis) versus the time of the X-ray signal measured by $D_{\text {ext }}$ (vertical axis), showing that the X-ray signal is also coincident with the time that the jet breaks apart. Timing when the plasma breaks apart is determined from images taken with an interframe time of $250 \mathrm{~ns}$, i.e., $4 \times 10^{6}$ frames per second. Lines shown are linear best fits according to a least square fit.

transient electrode voltage jump, and visual observation of the jet apparent breaking (i.e., dimming of visible light). Figures 3(d) and 3(h) in Ref. 22 provide a more definite definition of the dimming. Figure 3(d) in Ref. 22 is a superimposed image of the jet with visible light in blue and EUV in red, whereas Fig. 3(h) in Ref. 22 directly below it is purely visible. Arrows in Fig. 3(h) in Ref. 22 pinpoint the locations in the RT region where visible emission of the jet dims in between consecutive frames. During this time, Fig. 3(d) in Ref. 22 shows a strong burst of EUV emitted from the same region. The association of visible dimming with EUV emission is a reproducible phenomena. The time when the plasma breaks apart, synonymous with the visible dimming, is determined using images taken with $250 \mathrm{~ns}$ interframe time, i.e., a capture rate of $4 \times 10^{6}$ FPS. Figure 7(b) here illustrates less time resolution than Fig. 7(a) due to the interframe time being much larger than the time between voltage data points. $D_{\text {ext }}$ occasionally detects multiple bursts, and these coincide closely with multiple high-voltage probe jumps, suggesting multiple events taking place in a single shot. It was not possible to image these multiple events using the high-speed camera because the events were too separated in time for the camera timing to bracket these multiple events. However, the camera frequently captured one of the multiple detected RT-instigated breaking events, and the time of the captured event image was simultaneous with an X-ray scintillator signal.

\section{B. Internal detector, $D_{\text {int }}$}

$D_{\text {int }}$ is made of seven one inch diameter scintillators, each individually wrapped by aluminum foil and then black electrical tape. The housing holding the scintillators is vacuum-tight which allows this detector to be mounted inside the vacuum chamber. There is approximately $35 \mathrm{~cm}$ between the scintillators and the vacuum chamber axis. Each of the seven scintillators is linked to its own photomultiplier 


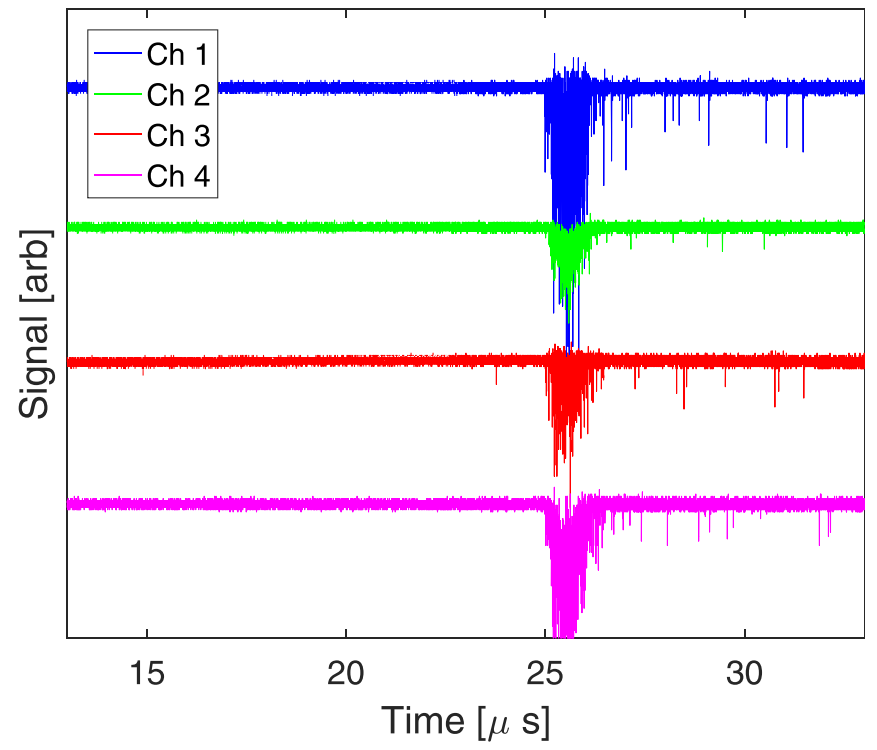

FIG. 8. Sample X-ray data taken by $D_{\text {int }}$. Four of the seven channels operate because the $1 \mathrm{GHz}$ oscilloscope only has four inputs. The vertical offset in each channel's data is chosen manually for presentation purposes. With no added offset, all the data prior to $25 \mu$ s would sit at the same vertical level. The figure shows that the signal envelopes from each channel are consistent with each other.

tube through a $2 \mathrm{~mm}$ diameter $10 \mathrm{~m}$ long optical fiber. The photomultiplier output is measured using a $1 \mathrm{GHz}$ bandwidth oscilloscope. This system has $5 \mathrm{~ns}$ time resolution. The oscilloscope has four channels.

Figure 8 shows data from channels $1,2,3$, and 4 of $D_{\text {int }}$. The signal envelopes show excellent agreement across channels. In the four datasets, there is no signal until $t \approx 25 \mu \mathrm{s}$ when all four channels detect an X-ray burst that lasts about $1.5 \mu \mathrm{s}$. Camera images from this shot show that the timing coincides with the jet breaking.
Figure 9 shows the X-ray data from channel 3 starting with a macroscopic look at the complete $15 \mu$ s dataset at the top of the figure. The middle plot in Fig. 9 shows a $1 \mu \mathrm{s}$ close-up of the X-rays. The bottom plot in Fig. 9 shows a $100 \mathrm{~ns}$ ultra-close-up of the data inside the dashed lines of the middle plot. This figure builds on the $D_{e x t}$ data by providing two important new pieces of information: (i) the X-ray signal consists of the scintillator collecting dozens to hundreds of discrete X-ray photons during the time when the jet is breaking and (ii) the X-rays are not mono-energetic as the observed photons have varying signal amplitudes.

\section{Amptek detector, $D_{\text {amptek }}$}

The Amptek XR100 Silicon Drift Detector, Damptek, detects X-rays in the $1-10 \mathrm{keV}$ range and has count rates as high as $10^{6}$ counts per second. From Sec. III B, X-ray illumination rates during the short timespan where $\mathrm{X}$-rays are detected are $\sim 100 / 1 \mu \mathrm{s}=10^{8}$ per second. From these count rates, it is apparent that $\mathrm{X}$-ray emission while the jet breaks is too fast for $D_{\text {amptek }}$ to detect individual photons, but $D_{\text {amptek }}$ is still useful qualitatively as a validation tool.

$D_{\text {amptek }}$ and $D_{\text {int }}$ were operated simultaneously on the experiment. The two detectors validated each other because when one detector saw an X-ray burst, the other did too. Equally as important, when one detector did not see an Xray signal, the other saw either no X-ray signal or an extremely weak one.

\section{X-ray camera, $D_{\text {CMos }}$}

$D_{C M O S}$ is a Mightex Systems MCE-B013-UW 1.3 Megapixel Windowless Camera with a sheet of aluminum foil placed in front of the CMOS sensor, a design that yields a reliable, energy-resolving $\mathrm{X}$-ray detector. $^{28-32}$ It is
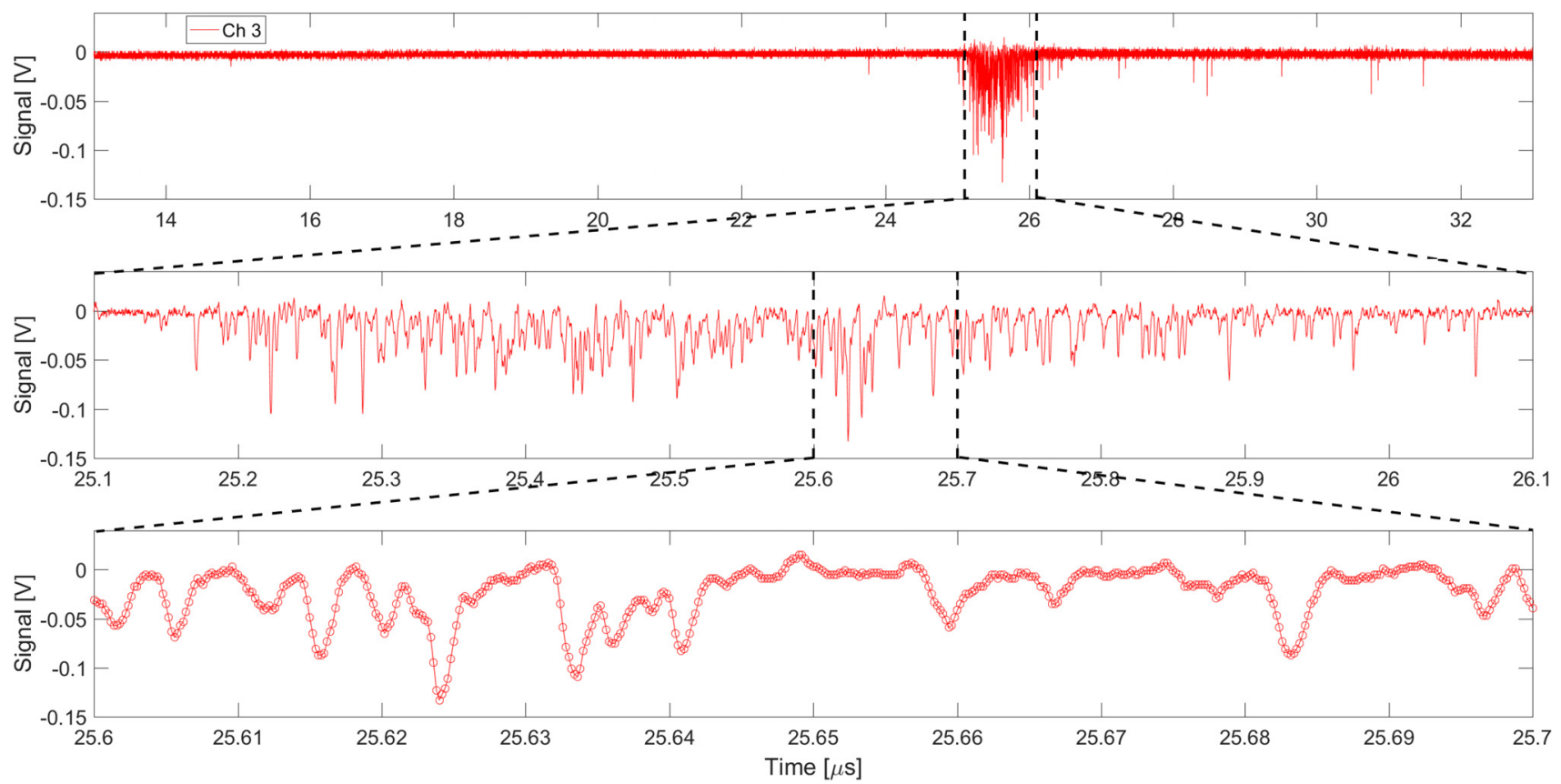

FIG. 9. The channel 3 data on the top row of this figure are the same data taken by $D_{\text {int }}$ that is shown as channel 3 of Fig. 8 . The middle plot shows a zoomedin view of $1 \mu$ s inside the dashed vertical lines from the top plot during the X-ray burst. The bottom plot shows a $100 \mathrm{~ns}$ snapshot, the region in the middle set of dashed lines blown up. 
important to note here that the term "X-ray" will be used as an all-encompassing term to describe ionizing radiation with energy $E>124 \mathrm{eV}$. X-rays and $\gamma$-rays are physically the same, the only distinction is in their origin: X-rays are from electrons outside the nucleus and from processes such as Bremsstrahlung, while $\gamma$-rays are from processes inside the nucleus. The windowless construction of the camera is important because most cameras are manufactured with a protective glass sheet glued directly on the CMOS sensor, and glass strongly attenuates $\mathrm{X}$-rays at the relevant energies. This glass sheet can be removed through careful application of heat to break the glue, ${ }^{28,31}$ but a simpler solution is to use a windowless camera. A single sheet of standard, storebought aluminum foil is $\sim 18 \mu \mathrm{m}$ thick. This foil transmits X-rays with energy $E>4 \mathrm{keV}$ (Ref. 27) while blocking visible light. Pixels impacted by X-rays have intensity proportional to energy deposited by the incoming photon, provided that the pixel is not saturated. A histogram of the pixel intensities gives the X-ray energy distribution which can be calibrated using X-ray sources with known energies. ${ }^{28-32}$

$D_{C M O S}$ was calibrated using an $87 \mu \mathrm{Ci}$ Co-57 sealed Mössbauer source and an $11 \mu \mathrm{Ci}$ Fe-55 stainless steel encapsulated source. Co-57 emits at 6.4, 14.4, and $122 \mathrm{keV}$, while $\mathrm{Fe}-55$ emits at $5.9 \mathrm{keV}$. It was observed that $\mathrm{X}$-ray photons incident on $D_{C M O S}$ deposit their energy in multiple neighboring pixels, hereby referred to as clusters, most commonly 2 or 3 pixels total. This seemed at first to contradict results found by Stoeckl et al. ${ }^{30}$ who reported that "a significant fraction of the X-ray photons deposit all their energy in one [13.5 $\mu \mathrm{m} \times 13.5 \mu \mathrm{m}]$ pixel." However, $D_{C M O S}$ has a pixel size of $5.2 \mu \mathrm{m} \times 5.2 \mu \mathrm{m}$, less than $1 / 6$ the area of a pixel in the sensor used by Stoeckl et al. More than $90 \%$ of the incident photons deposit their energy in 6 or fewer pixels, suggesting a similar effective deposition area to that in Stoeckl et al. To account for deposition in neighboring pixels, we developed a clustering algorithm where neighboring pixels are grouped into a single event. From an initial pixel with an intensity greater than 30 , an energy far above the measured background, neighboring pixels are searched for pixels with intensity greater than 12 , an intensity greater than $99 \%$ of the measured background. If a valid pixel is found, the neighbors of the new pixel are added to the search. All neighboring pixels with intensity greater than 12 are grouped into a single event with the initial pixel. A sample cluster from a Co-57 $\mathrm{X}$-ray is shown in Fig. 10.

The energies from this calibration are displayed in Fig. 11. The blue data are made up of $155000 \mathrm{X}$-rays detected from the $87 \mu \mathrm{Ci}$ Co-57 sealed Mössbauer source in 2000 images. The peaks, accented with blue vertical dashed lines, are assigned using known Co-57 emission energies: the upper peak is the $14.4 \mathrm{keV} \mathrm{X}$-ray emission and the lower peak is the $6.4 \mathrm{keV} \mathrm{K} \alpha$ line of a decay product. A histogram of 54000 photons detected from the $11 \mu \mathrm{Ci}$ Fe-55 sealed source in 2000 images is superimposed on the same axes. Using the calibration from the Co-57 histogram and assuming a linear relationship between the pixel intensity and energy deposited in a pixel, the orange peak from the Fe-55 data is calculated to be $5.9 \mathrm{keV}$, an exact match with the $\mathrm{Fe}-55$ emission.

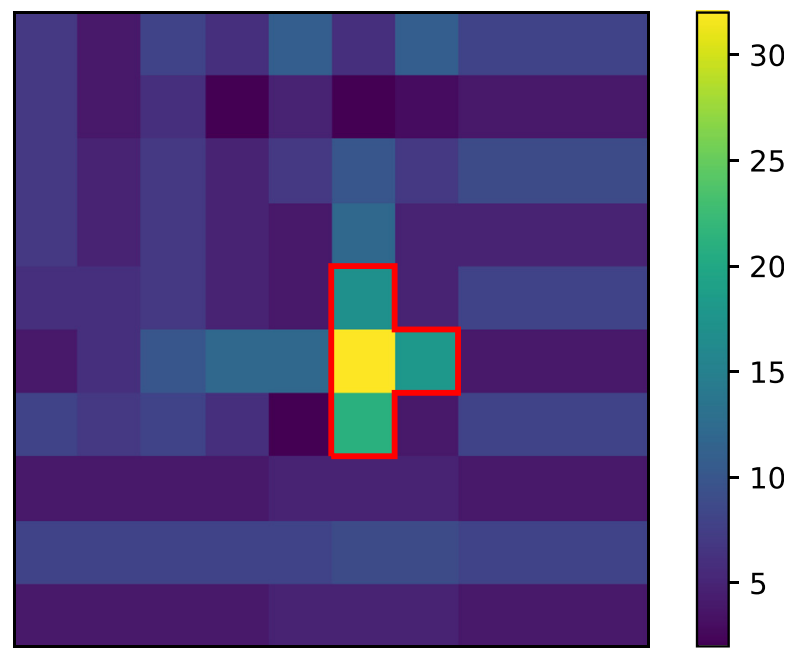

FIG. 10. A partial image from $D_{C M O S}$, showing a typical energy deposit on the camera sensor. This particular image is a Co-57 X-ray. The four pixels that contain the energy deposited from the X-ray are outlined in red. The yellow pixel contains the largest deposited energy. The pixel intensity of $\sim 30$ is much larger than where no interaction has occurred and thus is easily recognized.

$D_{C M O S}$ is mounted outside the vacuum chamber with the same orientation as $D_{\text {ext }}$. Because $D_{C M O S}$ is not a fast camera, it is triggered before the plasma shot and exposed during the whole duration of the shot. One image is obtained per shot. As a complement to $D_{\text {ext }}, D_{C M O S}$ is calibrated using known X-ray energies, and so, it directly measures the energy deposited by X-rays emitted from the jet. Figure 12 is a histogram that shows the X-ray energies. The blue data labeled "1 layer" shows the X-rays from 50 shots when only a single sheet of aluminum foil is placed in front of the camera sensor. This single sheet has the duty of blocking all visible light while passing X-rays. A distribution of energy of

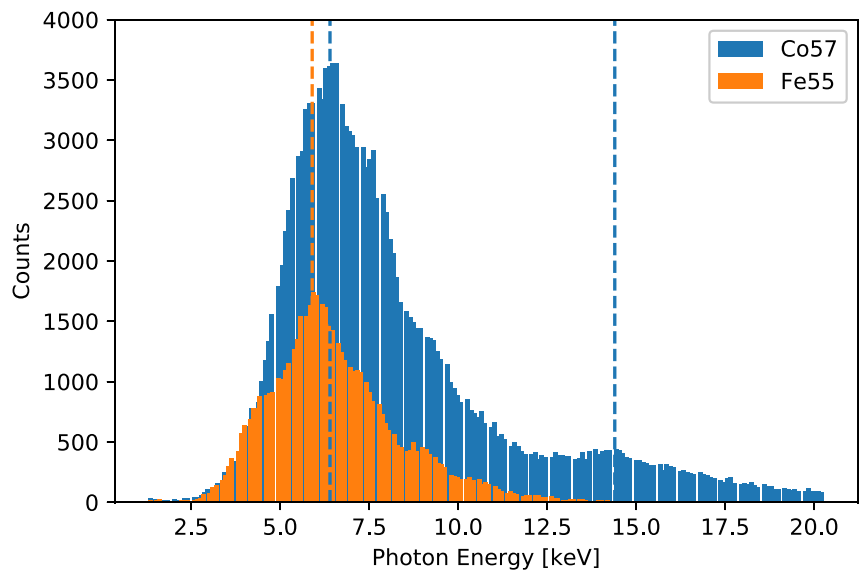

FIG. 11. Histograms of detected X-ray energies from an $87 \mu \mathrm{Ci} \mathrm{Co}-57$ sealed Mössbauer source (blue) and an $11 \mu \mathrm{Ci}$ sealed Fe-55 source (orange) using $D_{C M O S}$. 2000 images are taken with $D_{C M O S}$ exposed to Co-57, resulting in the detection of approximately $155000 \mathrm{X}$-ray photons, and 54000 X-ray photons are detected during 2000 frame exposure to the Fe-55 source. Dashed vertical lines highlight the peaks of the respective sources. The two peaks from Co-57 are fitted to known data, $6.4 \mathrm{keV}$ for the lower and $14.4 \mathrm{keV}$ for the upper. By assuming a linear relationship between energy deposited and pixel intensity, the peak associated with the Fe-55 source is calculated to be at $5.9 \mathrm{keV}$, an exact match with the documented Fe-55 emission 


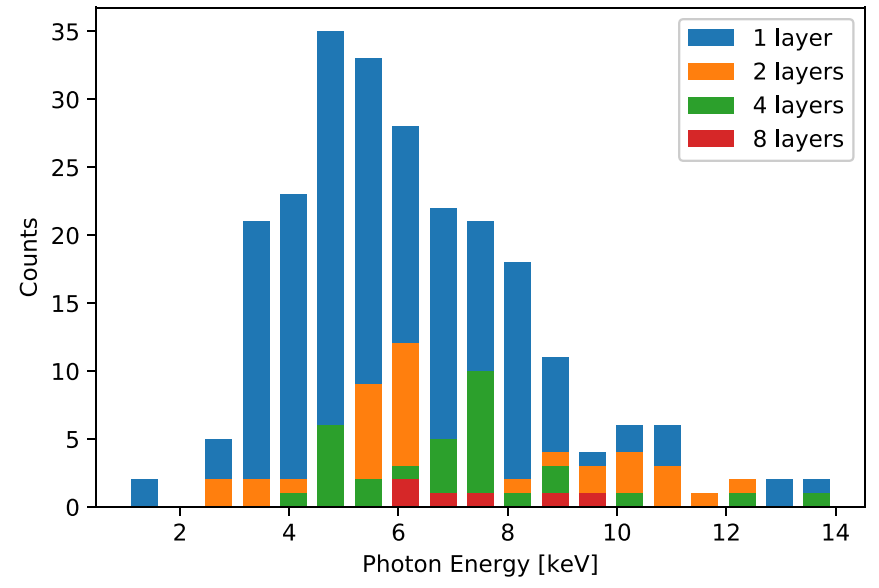

FIG. 12. Histograms of X-ray energies from the Caltech jet experiment. Each individual histogram is X-ray detection with a different thickness of the attenuating aluminum foil between $D_{C M O S}$ and the jet. The number of foil sheets includes the single sheet required for $D_{C M O S}$ to function as an $\mathrm{X}$-ray detector, i.e., there are $0,1,3$, and 7 layers of the additional attenuation material added for attenuation for each spectrum, respectively. Each spectrum includes $\mathrm{X}$-rays seen during plasma 50 shots. The energy spectrum is in excellent agreement with the findings from $D_{\text {ext }}$.

the X-rays is shown where the majority have energy between $4 \mathrm{keV}$ and $9 \mathrm{keV}$. The $5.8 \mathrm{keV}$ energy estimate from $D_{\text {ext }}$ shows excellent agreement with $D_{C M O S}$.

Aluminum foil is sequentially added in front of $D_{C M O S}$ to illustrate how the spectrum of detected photons changes with attenuation. Like the blue data in Fig. 12, each subsequent spectrum is also made up of data from 50 shots. As foil is added, the spectrum amplitude in Fig. 12 decreases as expected. Also as expected, the foil appears to attenuate a larger fraction of the incident X-ray photons with lower energy.

\section{MAGNETIC FIELD MEASUREMENTS}

The magnetic field evolution has been measured by an axially translatable radial B-dot probe array ${ }^{33-35}$ that records $B_{r}, B_{\phi}$, and $B_{z}$ at 11 radial locations spaced $2 \mathrm{~cm}$ apart. The probe is aligned normal to the electrode perimeter so that the respective $r, \phi$, and $z$ directions of the probe coils correspond to the chamber $r, \phi$, and $z$ coordinates. Figure 1 shows the magnetic probe array oriented such that it is positioned above the center of the electrodes.

By translating the probe through a sequence of $z$ positions for a sequence of plasma shots and measuring $B_{r}, B_{\phi}$, and $B_{z}$ at the 11 radial locations, an $r-z$ plane view of the magnetic field can be constructed. The configuration has high reproducibility before the kink starts, but during the kink and RT instabilities, the plasma has poor reproducibility. At later times, reproducibility is partially restored.

At each axial position $z$, data from 10 shots are averaged to give $\vec{B}\left(r, z=z_{\text {probe }}, t\right)$ for fixed $z_{\text {probe }}$. Thus, the measurement indicates an ensemble average. The probe is translated by $\Delta z=2.5 \mathrm{~cm}$, and the process is repeated. The final $110-$ point dataset is $\vec{B}(r, z, t)$ for grid points $r=0,2,4, \ldots, 20 \mathrm{~cm}$ and $z=7.5,10,12.5, \ldots, 30 \mathrm{~cm}$. The measurements are recorded with $10 \mathrm{~ns}$ resolution by 100 megasample per second digitizers. The electrode location defines $z=0$, and the poloidal flux is defined as

$$
\psi(r, z, t)=\int_{0}^{r} B_{z}\left(r^{\prime}, z, t\right) 2 \pi r^{\prime} d r^{\prime}
$$

which can be discretized as

$$
\psi(r, z, t)=\Delta r \sum_{r^{\prime}=0}^{r^{\prime}=r} B_{z}\left(r^{\prime}, z, t\right) 2 \pi r^{\prime},
$$

where $\Delta r=2 \mathrm{~cm}$ is the radial distance between stations in the B-dot probe array. One key assumption that is evident from this integration is that the ensemble average magnetic field measurement $B(r, z, t)$ is constant in the toroidal $\hat{\phi}$ direction. The poloidal current is defined as

$$
I(r, z, t)=\frac{2 \pi}{\mu_{0}} r B_{\phi}(r, z, t) .
$$

The top row of Fig. 13 shows camera frames from 5 to $35 \mu s$. These images show that the jet flows without kinking until about 15-20 $\mu$ s and that the RT occurs at 25-30 $\mu$ s. The second from top row shows the corresponding evolution of $\psi(r, z, t)$, and the third from top row shows the evolution of $I(r, z, t)$. Once the jet propagates past $z=7.5 \mathrm{~cm}$, the magnetic probe array registers a signal, and from 7.5 to $20 \mu \mathrm{s}$, jet propagation in the $+z$ direction is clearly evident from both the camera frames and the $I, \psi$ plots. This initial segment of the jet evolution has high reproducibility.

The next phase starts at the onset of the kink instability which is then followed by the RT instability breaking the jet. The exact timing varies from shot to shot as is shown in Fig. 7. For the particular photograph sequence shown in the top row of Fig. 13, the RT instability starts at $\sim 25 \mu \mathrm{s}$. Figure 13 shows that the flux surfaces become irregular with reduced shot-to-shot reproducibility from 25 to approximately $35 \mu \mathrm{s}$. It is at times in this interval that X-rays are observed (bottom row).

Figure 13 shows that the magnetic flux profile changes abruptly at the time of RT. After the RT, X-rays, and other associated simultaneous phenomena, the $\psi$ profile differs from its prior profile. There is also evidence from the third row of Fig. 13 that the shot-averaged $I(r, z, t)$ transiently goes to zero at certain axial locations which indicates a break in the shot-averaged $J_{z}$ circuit. Observation of the changing magnetic field at the spatial and temporal scale of the RT ripples is not possible because the spatial scale of these ripples is smaller than the probe spatial resolution and because the location of the RT instability varies from shot to shot.

\section{PHENOMENA SIMULTANEOUS WITH RT INSTABILITY}

The combined observation of eight different simultaneous phenomena at the time of the RT instability provides strong circumstantial evidence that the RT instigates an anomalous event that is outside the scope of ideal MHD. These phenomena are as follows:

1. The high speed camera shows that the jet appears to undergo a RT instability and then break apart. ${ }^{23}$

2. There is a transient EUV burst ${ }^{22}$ at the RT location. 


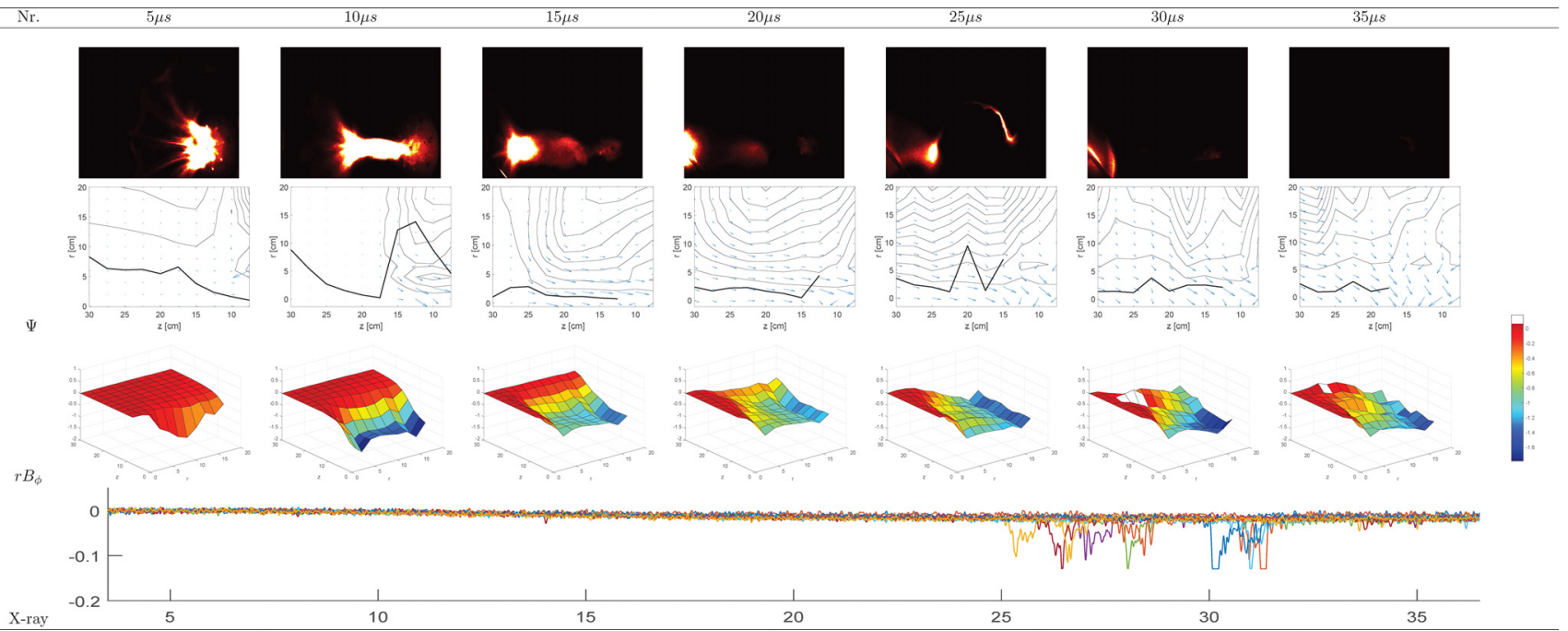

FIG. 13. Timing relationships for camera images, poloidal magnetic flux, poloidal current, and X-rays. The top row shows false color images of the plasma taken every $5 \mu \mathrm{s}$ using a high-speed camera. The plasma jet is clearly seen propagating down the chamber from right to left as time increases from 5 to $20 \mu \mathrm{s}$. The kink and RT instabilities break apart the plasma at $t \approx 25 \mu \mathrm{s}$ in this set of images. The second row (denoted $\psi$ ) shows poloidal flux contours calculated from magnetic probe measurements using Eq. (2) for times corresponding to the photos in the top row (the black line is where $B_{\phi}=0$ ). As in the photographs, the poloidal flux surfaces are clearly stretching from right to left during the 5 to $20 \mu$ s interval, coincident with the jet traveling down the chamber. After the RT instability breaks apart the plasma, the flux surfaces change substantially. The third row of images (denoted $r B_{\phi}$ ) shows the poloidal current in arbitrary units. The colorbar on the right-hand side applies to all seven plots. The jet right to left motion from 5 to $20 \mu$ s can also be seen clearly in these plots. After 20 $\mu \mathrm{s}$, the poloidal current surfaces become more random, and there are locations where the current vanishes or changes the sign, i.e., there is a current disruption. The sign change is highlighted by the white color in the colormap which makes it easy to observe. The bottom plot is stretched over all 7 columns and shows $\mathrm{X}$-ray scintillator signals for 10 of the 100 shots used to make the poloidal flux and poloidal current plots in the second and third rows. The $10 \mathrm{X}$-ray signals occur at times $25 \mu \mathrm{s}<t<32 \mu \mathrm{s}$, and Fig. 7 shows that the X-ray signals are coincident with the RT instability and voltage jump. The temporal irreproducibility of the RT signal and associated phenomena is evident from the scatter in the times for the $10 \mathrm{X}$-ray signals. This scatter indicates that the magnetic plots must be considered as an ensemble average of the poloidal flux and current over many shots.

3. The spectrometer shows ${ }^{22}$ Ar II line emission before the RT instability but Ar III and Ar IV emission during the RT instability; this indicates electron heating.

4. An rf magnetic probe indicates a burst of high frequency waves ${ }^{22}$ tentatively identified as whistlers.

5. The spectrometer shows ${ }^{22}$ increased Doppler broadening of the ion lines; this indicates ion heating.

6. The magnetic probe array shows that the magnetic field morphology after breaking is substantially different from before.

7. The high voltage probe shows a $500-1000 \mathrm{~V}$ jump.

8. There is a transient burst of X-rays.

\section{ENERGY AND ELECTRIC FIELD ESTIMATION}

The energy in the capacitor bank driving the jet is $W_{c}$ $=C V^{2} / 2=120 \mu \mathrm{F} \times(5 \mathrm{kV})^{2} / 2=1.5 \mathrm{~kJ}$. The thermal energy in the particles in the breaking region is $W_{t h}=n V k_{b} T$, where $n$ is the particle density, $V$ is the volume of the region assumed to be a cylinder, and $T$ is the temperature. Assuming a nominal radius $r=2 \mathrm{~cm}$ and length $l=0.4$ $\mathrm{m}$, this volume is $V=\pi r^{2} L=5 \times 10^{-4} \mathrm{~m}^{3}$. Using the nominal density $n=3 \times 10^{22} \mathrm{~m}^{-3}$ from the laser interferometer ${ }^{25}$ and temperature $T=2 \mathrm{eV}$, the thermal energy in the particles is $W_{t h}=n V k_{b} T=5 \mathrm{~J}$. The circuit magnetic energy at the time of the X-ray burst is $W_{\text {mag }}=L I^{2} / 2=54 \mathrm{nH}$ $\times(60 \mathrm{kA})^{2} / 2=100 \mathrm{~J}$. Calibration of $D_{\text {ext }}$ using X-rays from a thorium reference source and taking into account the solid angle subtended by $D_{e x t}$ indicate that the radiated X-ray energy is $\sim 10^{-8} \mathrm{~J}$, indicating that only a tiny fraction of the electrons emit X-rays. This imposes the important constraint that any proposed mechanism for accelerating electrons to high energy in a collisional plasma should work on only a tiny fraction of the electrons.

Since argon's K-shell energy is $3.2 \mathrm{keV},{ }^{36}$ atomic line radiation cannot explain the observed $\sim 6 \mathrm{keV}$ photons. As additional evidence that the radiation is not from Argon's Kshell, Fig. 12 presented in Sec. III D shows that the energy of the emitted photons from the plasma is not mono-energetic or at multiple specific lines.

This elimination of $\mathrm{K}$-shell radiation suggests Bremsstrahlung to be the likely mechanism. A $6 \mathrm{keV}$ electron travels at $v \approx 5 \times 10^{7} \mathrm{~m} / \mathrm{s}$. The X-ray pulse lasts approximately $1 \mu \mathrm{s}$ as seen in the top trace in Fig. 4. If a $2 \mathrm{eV}$ electron uniformly accelerates to $6 \mathrm{keV}$ in $0.5 \mu \mathrm{s}$, an electric field $E=520 \mathrm{~V} / \mathrm{m}$ would be required and the electron would travel $11 \mathrm{~m}$. If the acceleration time constraint was doubled to $1 \mu \mathrm{s}$, the acceleration would result in the electron traveling twice the distance: $22 \mathrm{~m}$. Such long acceleration distances are not credible because the high-speed imaging shows that the breaking region has a scale length of $\sim 10 \mathrm{~cm}$. If, instead, the electron reaches $6 \mathrm{keV}$ in $10 \mathrm{~cm}$, then an $E=6 \times 10^{4} \mathrm{~V} /$ $\mathrm{m}$ electric field is required and the acceleration of an individual electron would take only $\sim 4 \mathrm{~ns}$ if it did not collide. This electric field is much smaller than the Dreicer ${ }^{37}$ electric field $E_{D}=5.6 \times 10^{-18} n_{e} Z T_{e}^{-1} \ln \Lambda=8 \times 10^{5} \mathrm{~V} / \mathrm{m}$, so the plasma would be collisional and not running away (i.e., not having all electrons accelerated to high energy).

Figure 8 shows that the X-ray signal consists of dozens to hundreds of discrete photons emitted throughout the 
$\sim 1 \mu$ s time interval when the jet is breaking. This suggests that electron acceleration continuously occurs during the entire $1 \mu \mathrm{s}$ interval. This multiplicity of photons is consistent with the acceleration of individual electrons in $4 \mathrm{~ns}$ over a $10 \mathrm{~cm}$ path by $E=6 \times 10^{4} \mathrm{~V} / \mathrm{m}$.

\section{DISCUSSION}

An inductive electric field could arise in a manner similar to (but with more extreme parameters) the spark that occurs when a toaster is unplugged from the wall; this is in effect an opening switch voltage source. ${ }^{38-40}$ When a toaster is unplugged, the circuit produces a large $L d I / d t$ voltage that attempts to keep the electric current flowing; here, $L$ is the inductance of the wiring up to the wall socket. This situation is also analogous to a log pile-up on a fast-moving river with the river flow velocity corresponding to current, the mass of the upstream river water to inductance, the momentum of this upstream water to magnetic flux, and the pressure drop across the log pile-up analogous to the voltage drop LdI/dt. As shown in Fig. 3(b), the RT instability breaking the jet acts like the opening switch because the RT instability chokes the current channel diameter $d$ to be smaller than the ion skin depth $c / \omega_{p i}$. When this happens, the electron drift velocity $v_{d}=J / n e$ becomes of the order of the Alfvén velocity $v_{A}=B / \sqrt{\mu_{0} n m_{i}}$. ${ }^{23}$ It is reasonable to presume that the plasma cannot conduct an electric current requiring such a large electron drift velocity; thus, the choke region behaves as an opening switch that interrupts the current and a voltage $L d I / d t$ appears across the gap. ${ }^{41-43}$ This is also like the pressure drop across the log pile-up in the river analogy.

The plasma and initial power supply electric circuit can be modeled as an $L C$ circuit, where $C$ is from the capacitor bank power supply and $L$ comes from both the plasma and the cabling up to the plasma. Figures 3(a) and 3(b) show this circuit diagram immediately before and after the RT instability breaks the plasma apart. When the circuit is closed, the $L C$ circuit quarter-cycle time is $t_{1 / 4}=\pi / 2 \sqrt{L C}$. The observed current rise-time is $\sim 4 \mu \mathrm{s}$, so using the $120 \mu \mathrm{F}$ of the fast capacitor bank gives $L \simeq 54 \mathrm{nH}$, most of which is in the path from the capacitor to where the circuit opens. The Pulse Forming Network (PFN) cables have a similar inductance. The electric current flowing in the jet at the time of the RT instability is $\sim 60 \mathrm{kA}$, and the circuit has $L=54 \mathrm{nH}$, so interrupting this circuit produces a voltage of $V=L d I / d t$ across the gap shown in Fig. 3(b). It is important to note that this inductive $L d I / d t$ voltage is not related to the voltage on the capacitor bank and could be much larger. Jiang et al. ${ }^{39}$ and Takaki et al..$^{40}$ discussed this in great detail.

The actual interruption of this current is not instantaneous and likely has shorter duration than the observed $\mathrm{X}$ ray burst. A nominal interruption time of $500 \mathrm{~ns}$ is chosen based upon growth rates for the RT instability from Moser. ${ }^{23}$ The direct estimation from the images in Ref. 23 yields $\gamma_{R T}=1 \times 10^{6} / \mathrm{s}$, and the calculation using the measured effective gravity and observed ripple wavelength gives $\gamma_{R T}=\sqrt{g_{\text {eff }}}=3 \times 10^{6} / \mathrm{s}$, where both $g_{\text {eff }}$ and $k$ come from the images in Ref. 23. Choosing a nominal interruption time intermediate between the observed and calculated growth rates, $500 \mathrm{~ns}$, will produce an inductive voltage of $V$ $=L d I / d t \simeq 6 \mathrm{kV}$; this is consistent with accelerating electrons to $\sim 6 \mathrm{keV}$. The value of $6 \mathrm{keV}$ is nominal because some electrons may be accelerated to higher energies while only emitting Bremsstrahlung photons of lower energy since their slowing-down collisions can be less than head-on.

The simultaneous substantive change in the global shotaveraged poloidal flux structure from before to after the RT instability, the X-ray evidence for a large transient electric field, and the six other measurements listed in Sec. V provide strong circumstantial evidence that a fast magnetic reconnection event is instigated by the RT instability. If one were to argue that there is no magnetic reconnection event, i.e., if one were to argue that magnetic flux remains frozen into the plasma frame throughout the time when the above eight phenomena occur, then there would be no electric field in the plasma frame and so no means for accelerating electrons to high energy. Furthermore, the voltage transient observed by the high voltage probe indicates that there is a sudden change in the magnetic flux linked by the electric circuit going from the inner electrode to the outer electrode. If magnetic flux were frozen into the plasma, then no such change in flux linked by this circuit could occur, and so, no voltage transient would be observed at the electrodes.

At this point, the questions from the introduction reemerge: (i) How does a small subset of particles get energized? (ii) How is the subset selected? (iii) Why can this subset accelerate while the plasma is both cold and collisional? We postulate the mechanism is as follows: The key ideas are the energy dependence of the mean free path and the statistical nature of the mean free path. The plasma is very dense and so there are a large number of electrons in the reconnection region. The reconnection electric field accelerates all the electrons, but because the plasma is very collisional with a mean free path initially on the order of $1 \mu \mathrm{m}$, only $e^{-1}$ of the electrons are successfully accelerated over the whole mean free path as $1-e^{-1}$ $\approx 0.66$ of the electrons are scattered. The ones that did not collide are then moving with more kinetic energy than initially, so their next mean free path is longer. Again, $e^{-1}$ are successfully accelerated and gain even more energy than previously. This cycle of acceleration and increasing mean free path repeats over the reconnection distance. This type of acceleration can not only explain the X-ray observation but also the EUV observation as particles that only accelerate part of the way before radiating could easily emit EUV. Because of the high density, a tiny but macroscopic number of initially thermal electrons can successfully accelerate to $\mathrm{keV}$ energies and then finally collide to emit a large energy photon. A full quantitative description of this proposed mechanism will be reported separately.

\section{CONCLUSION}

An X-ray burst with a spectrum of energies on the order of $6 \mathrm{keV}$ is observed to be emitted from a cold, collisional MHD-driven plasma jet when it undergoes a kink-instigated RT instability. The burst has been detected by four different detectors: $D_{\text {ext }}, D_{\text {int }}, D_{\text {amptek }}$, and $D_{C M O S}$. Combined data from all 4 detectors show that the burst consists of dozens to 
hundreds of non-mono-energetic X-ray photons emitted in the direction of the detector over a timespan of approximately $1 \mu \mathrm{s}$.

The X-ray burst is correlated in time with magnetic data that show a clear change in the magnetic topology of the system. Six other diagnostics show abrupt changes at the same time. The high speed camera shows the jet undergoing a kink, a Rayleigh-Taylor instability, and then breaking apart, EUV optics image a transient EUV burst, electron and ion heating takes place, a burst of high frequency waves tentatively labeled as whistlers is seen, and a high voltage probe shows a $500-1000 \mathrm{~V}$ jump.

These eight measurements are consistent with the explanation that a fast 3-D magnetic reconnection event results from the current in the jet being choked down and disrupted. The proposed statistical avalanching acceleration mechanism explains these observations and is likely relevant to solar and astrophysical plasmas.

\section{ACKNOWLEDGMENTS}

We would like to thank Professor Gunsu S. Yun of Pohang University of Science and Technology in Pohang, South Korea, for bringing and operating $D_{\text {amptek }}$ on the jet experiment at Caltech. $D_{\text {amptek }}$ provided valuable qualitative results.

This material is based upon work supported by the U.S. Department of Energy Office of Science, Office of Fusion Energy Sciences, under Award No. DE-FG02-04ER54755 and by the Air Force Office of Scientific Research under Award No. FA9550-11-1-0184. Experimental data are available at https://data.caltech.edu/records/305.

${ }^{1}$ P. C. Thonemann, E. P. Butt, R. Carruthers, A. N. Dellis, D. W. Fry, A. Gibson, G. N. Harding, D. J. Lees, R. W. P. McWhirter, R. S. Pease, S. A. Ramsden, and S. Ward, "Production of higher temperatures and nuclear reactions in a gas discharge," Nature 181(4604), 217-220 (1958).

${ }^{2}$ S. A. Colgate and H. P. Furth, "Stabilized pinch and controlled fusion power," Science 128(3320), 337-343 (1958).

${ }^{3}$ B. Rose, A. E. Taylor, and E. Wood, "Measurement of the neutron spectrum from ZETA," Nature 181(4624), 1630-1632 (1958).

${ }^{4}$ L. Spitzer, "Co-operative phenomena in hot plasmas," Nature 181(4604), 221-222 (1958).

${ }^{5} \mathrm{~K}$. A. George, "Neutron production and temperature in ZETA," Nature 182(4637), 745-746 (1958).

${ }^{6}$ W. H. Bostick, V. Nardi, and W. Prior, "Production and confinement of high-density plasmas," Ann. N. Y. Acad. Sci. 251, 2-29 (1975).

${ }^{7}$ V. A. Gribkov, B. Bienkowska, M. Borowiecki, A. V. Dubrovsky, I. Ivanova-Stanik, L. Karpinski, R. A. Miklaszewski, M. Paduch, M. Scholz, and K. Tomaszewski, "Plasma dynamics in pf-1000 device under fullscale energy storage: I. Pinch dynamics, shock-wave diffraction, and inertial electrode," J. Phys. D: Appl. Phys. 40(7), 1977-1989 (2007).

${ }^{8}$ R. E. Chrien, J. C. Fernandez, I. Henins, R. M. Mayo, and F. J. Wysocki, "Evidence for runaway electrons in a spheromak plasma," Nucl. Fusion 31(7), 1390-1393 (1991).

${ }^{9}$ R. D. Gill, "Generation and loss of runaway electrons following disruptions in JET," Nucl. Fusion 33(11), 1613-1625 (1993).

${ }^{10}$ S. G. Benka and G. D. Holman, "A thermal/nonthermal model for solar hard x-ray-bursts," Astrophys. J. 435(1), 469-481 (1994).

${ }^{11}$ J. A. Miller, P. J. Cargill, A. G. Emslie, G. D. Holman, B. R. Dennis, T. N. LaRosa, R. M. Winglee, S. G. Benka, and S. Tsuneta, "Critical issues for understanding particle acceleration in impulsive solar flares," J. Geophys. Res.: Space Phys. 102(A7), 14631-14659, https://doi.org/10.1029/ 97JA00976 (1997).

${ }^{12}$ Y. E. Litvinenko, "Electron acceleration in solar flares," in Energy Release and Particle Acceleration in the Solar Atmosphere-Flares and
Related Phenomena, Advances in Space Research, 2nd World Space Congress/34th COSPAR Scientific Assembly, Houston, Texas, 10-19 October 2002, edited by B. R. Dennis, T. Kosugi, and R. P. Lin (Pergamon-Elsevier Science LTD, 2003), Vol. 32, pp. 2385-2391.

${ }^{13}$ M. J. Aschwanden, Physics of the Solar Corona (Springer-Verlag, Berlin/ Heidelberg, 2005).

${ }^{14}$ V. Petrosian, "Stochastic acceleration by turbulence," Space Sci. Rev. 173(1-4), 535-556 (2012).

${ }^{15} \mathrm{~K}$. Jun, "High energy observations of AGN jets and their future prospects," in Accelerators in the Universe, Conference on Accelerators in the Universe, Tsukuba, Japan, 12-14 March 2008, edited by H. Kodama and K. Ioka [AIP Conf. Proc. 1040, 191-205 (2008)].

${ }^{16}$ B. S. Hogan, M. L. Lister, P. Kharb, H. L. Marshall, and N. J. Cooper, "Chandra discovery of 10 new x-ray jets associated with FR II radio coreselected AGNs in the mojave sample," Astrophys. J. 730(2), 92 (2011)

${ }^{17}$ A. Gibson, "Possibility of ion runaway in ZETA," Nature 183(4654), 101-102 (1959).

${ }^{18}$ E. N. Parker and D. A. Tidman, "Suprathermal particles," Phys. Rev. 111(5), 1206-1211 (1958).

${ }^{19}$ D. E. McKenzie, "Observational studies of reconnection in the solar corona," Phys. Plasmas 18(11), 111205 (2011).

${ }^{20}$ A. Stanier, P. Browning, and S. Dalla, "Solar particle acceleration at reconnecting 3D null points," Astron. Astrophys. 542, A47 (2012).

${ }^{21}$ V. V. Zharkova, K. Arzner, A. O. Benz, P. Browning, C. Dauphin, A. G. Emslie, L. Fletcher, E. P. Kontar, G. Mann, M. Onofri, V. Petrosian, R. Turkmani, N. Vilmer, and L. Vlahos, "Recent advances in understanding particle acceleration processes in solar flares," Space Sci. Rev. 159(1-4), 357-420 (2011).

${ }^{22}$ K.-B. Chai, X. Zhai, and P. M. Bellan, "Extreme ultra-violet burst, particle heating, and whistler wave emission in fast magnetic reconnection induced by kink-driven Rayleigh-Taylor instability," Phys. Plasmas 23(3), 032122 (2016).

${ }^{23}$ A. L. Moser and P. M. Bellan, "Magnetic reconnection from a multiscale instability cascade," Nature 482(7385), 379-381 (2012).

${ }^{24}$ S. C. Hsu and P. M. Bellan, "A laboratory plasma experiment for studying magnetic dynamics of accretion discs and jets," Mon. Not. R. Astron. Soc. 334(2), 257-261 (2002).

${ }^{25}$ D. Kumar and P. M. Bellan, "Heterodyne interferometer with unequal path lengths," Rev. Sci. Instrum. 77(8), 083503 (2006).

${ }^{26}$ S. C. Hsu and P. M. Bellan, "Experimental identification of the kink instability as a poloidal flux amplification mechanism for coaxial gun spheromak formation," Phys. Rev. Lett. 90(21), 215002 (2003).

${ }^{27}$ B. L. Henke, E. M. Gullikson, and J. C. Davis, "X-ray interactions: Photoabsorption, scattering, transmission, and reflection at $E=50-30,000$ ev, $Z=1-92$," At. Data Nucl. Data Tables 54(2), 181-342 (1993).

${ }^{28}$ R. G. van Silfhout and A. S. Kachatkou, "Fibre-optic coupling to highresolution CCD and CMOS image sensors," Nucl. Instrum. Methods Phys. Res., Sect. A 597(2-3), 266-269 (2008).

${ }^{29}$ F. Nachtrab, T. Hofmann, M. Firsching, N. Uhlmann, and R. Hanke, "Simple solutions for spectroscopic, photon counting X-ray imaging detectors," in IEEE Nuclear Science Symposium and Medical Imaging Conference, 2009 IEEE Nuclear Science Symposium Conference Record, Orlando, FL, 25-31 October 2009, edited by B. Yu (IEEE, 2009), Vols. $1-5$, p. 1636.

${ }^{30}$ C. Stoeckl, W. Theobald, T. C. Sangster, M. H. Key, P. Patel, B. B. Zhang, R. Clarke, S. Karsch, and P. Norreys, "Operation of a single-photon-counting $\mathrm{X}$-ray charge-coupled device camera spectrometer in a petawatt environment," in 15th Topical Conference on High-Temperature Plasma Diagnostics, San Diego, CA, 19-22 April 2004 [Rev. Sci. Instrum. 75(10), 3705-3707 (2004)].

${ }^{31}$ D. W. Lane, "X-ray imaging and spectroscopy using low cost COTS CMOS sensors," Nucl. Instrum. Methods Phyics Res., Sect. B 284, 29-32 (2012).

${ }^{32}$ H. G. Kang, J.-J. Song, K. Lee, K. C. Nam, S. J. Hong, and H. C. Kim, "An investigation of medical radiation detection using CMOS image sensors in smartphones," Nucl. Instrum. Methods Phys. Res., Sect. A 823, 126-134 (2016).

${ }^{33}$ M. Auna Louise, "Dynamics of magnetically driven plasma jets: An instability of an instability, gas cloud impacts, shocks, and other deformations," Ph.D. thesis (California Institute of Technology, 2012).

${ }^{34}$ C. A. Romero-Talamas, P. M. Bellan, and S. C. Hsu, "Multielement magnetic probe using commercial chip inductors," Rev. Sci. Instrum. 75(8), 2664-2667 (2004). 
${ }^{35}$ J. Yee and P. M. Bellan, "Taylor relaxation and lambda decay of unbounded, freely expanding spheromaks," Phys. Plasmas 7(9), 3625-3640 (2000).

${ }^{36}$ R. D. Deslattes, E. G. Kessler, Jr., P. Indelicato, L. de Billy, E. Lindroth, J. Anton, J. S. Coursey, D. J. Schwab, C. Chang, R. Sukumar, K. Olsen, and R. A. Dragoset, https://physics.nist.gov/cgi-bin/XrayTrans/search.pl?element= Ar\&lower=\&upper=\&units=eV for "X-ray transition energies (version 1.2)," 2005; accessed 5 December 2016.

${ }^{37} \mathrm{H}$. Dreicer, "Electron and ion runaway in a fully ionized gas. 1," Phys. Rev. 115(2), 238-249 (1959).

${ }^{38}$ K. H. Schoenbach, M. Kristiansen, and G. Schaefer, "A review of opening switch technology for inductive energy-storage," Proc. IEEE 72(8), 1019-1040 (1984).

${ }^{39}$ W. Jiang, K. Nakahiro, K. Yatsui, J.-H. Kim, and N. Shimizu, "Repetitive pulsed high voltage generation using inductive energy storage with static-induction thyristor as opening switch," IEEE Trans. Dielectr. Electr. Insul. 14(4), 941-946 (2007).

${ }^{40}$ K. Takaki, K. Kanesawa, S. Mukaigawa, T. Fujiwara, and T. Go, "Energy efficiency of corona discharge reactor driven by inductive energy storage system pulsed power generator," IEEE Trans. Dielectr. Electr. Insul. 14(4), 834-845 (2007).

${ }^{41}$ C. Jacobsen and P. Carlqvist, "Solar flares caused by circuit interruptions," Icarus 3(3), 270 (1964).

${ }^{42} \mathrm{H}$. Alfvén and P. Carlqvist, "Currents in the solar atmosphere and a theory of solar flares," Sol. Phys. 1(2), 220-228 (1967).

${ }^{43}$ R. L. Stenzel, W. Gekelman, and N. Wild, "Magnetic-field line reconnection experiments. 5. Current disruptions and double-layers," J. Geophys. Res.: Space Phys. 88(NA6), 4793-4804, https://doi.org/10.1029/ JA088iA06p04793 (1983). 